\title{
Expression profiles of long non-coding RNAs in the cartilage of patients with knee osteoarthritis and normal individuals
}

\author{
YANCHANG LIU, JUEHUA JING, HAORAN YU, JISEN ZHANG, QILIANG CAO, \\ XIN ZHANG, JIANJUN LIU, SHUO ZHANG and WENDAN CHENG \\ Department of Orthopedic Surgery, The Second Affiliated Hospital of \\ Anhui Medical University, Hefei, Anhui 230601, P.R. China
}

Received May 29, 2019; Accepted December 12, 2019

DOI: $10.3892 /$ etm.2021.9796

\begin{abstract}
Knee osteoarthritis is caused by a multifactorial imbalance in the synthesis and degradation of knee chondrocytes, subchondral bone and extracellular matrix. Abnormal expression of long non-coding RNAs (lncRNAs) affects the metabolism, synovitis, autophagy and apoptosis of chondrocytes, as well as the production of cartilage matrix. The aim of the present study was to identify novel targets for the treatment of osteoarthritis and to examine the pathogenesis of the disease. The lncRNA expression profiles of seven patients with knee osteoarthritis and six healthy controls were examined by RNA-sequencing. Differentially expressed IncRNAs were selected for bioinformatics analyses, including Gene Ontology (GO) and Kyoto Encyclopedia of Genes and Genomes (KEGG) pathway enrichment. Reverse transcription-quantitative PCR (RT-qPCR) was used to further investigate the differential expression of the lncRNAs. A total of 23,583 lncRNAs were identified in osteoarthritis cartilage, including 5,255 upregulated and 5,690 downregulated IncRNAs, compared with normal cartilage. Although there were more downregulated lncRNAs compared with upregulated lncRNAs, among the changed lncRNAs (fold-change $>6$ ), there were more upregulated lncRNAs compared with downregulated lncRNAs. Several lncRNAs exhibiting differences were identified as potential therapeutic targets
\end{abstract}

Correspondence to: Mr. Wendan Cheng, Department of Orthopedic Surgery, The Second Affiliated Hospital of Anhui Medical University, 678 Fu Rong Road, Hefei, Anhui 230601, P.R. China

E-mail: sunyccc@126.com

Abbreviations: lncRNA, long non-coding RNA; NC, normal group; OA, knee osteoarthritis group; RT-qPCR, reverse transcription-quantitative PCR; GO, Gene Ontology; KEGG, Kyoto Encyclopedia of Genes and Genomes; ITGA5, integrin- $\alpha 5 ; \mathrm{H}$ MGB1, high mobility group protein B1; IFITM3; interferon induced transmembrane protein 3

Key words: knee osteoarthritis, long non-coding RNAs, osteoarthritis pathogenesis, RNA-sequencing in knee osteoarthritis. GO and KEGG pathway analyses were performed for the target genes of the differentially expressed lncRNAs. RT-qPCR validation was performed on three randomly selected upregulated and downregulated lncRNAs. The results of RT-qPCR were consistent with the findings obtained by RNA-sequencing analysis. The findings from the present study may contribute to the diagnosis of osteoarthritis and may predict the development of osteoarthritis. Furthermore, the differentially expressed lncRNAs may aid in the identification of novel candidate targets for the treatment of knee osteoarthritis.

\section{Introduction}

Knee osteoarthritis is the most common chronic degenerative bone and joint disease in middle-aged and elderly individuals worldwide (1). Knee osteoarthritis is a heterogeneous disease with an incidence of $\sim 3 \%(2,3)$, and results from a multifactorial imbalance in the synthesis and degradation of knee chondrocytes subchondral bone and extracellular matrix (4). Abnormal joint metabolism leads to degeneration of knee articular cartilage and subchondral bone, joint fiber hyperplasia, synovial non-specific inflammation and other lesions (5). Due to the continuous damage caused by chronic inflammation and the progressive structural changes of the knee joint tissue, the disease progresses continuously (6). An irreversible loss of knee function and pain are among the common causes of decreased quality of life and disability associated with knee osteoarthritis (7).

The main clinical manifestations of knee osteoarthritis are slow-developing knee pain, tenderness, stiffness, joint swelling, limited mobility and knee deformity (8). The occurrence and development of knee osteoarthritis are the result of the interaction between biomechanical and biological factors, including the mechanical environment around the knee joint, the metabolic rate of the individual, the apoptosis of chondrocytes and the levels of related cytokines, such as interleukin (IL) IL-4, IL-10 and tumor necrosis factor (TNF)- $\alpha$ (9-11). A number of risk factors for knee osteoarthritis have been identified, including age, sex, obesity, inflammation, strain, trauma and genetics (12-14). The development and progression of knee osteoarthritis is complex and at present, effective 
treatment strategies and clear therapeutic targets have not been identified (15).

Long non-coding RNAs (lncRNAs) are RNA molecules $>200$ nucleotides in length that are the by-product of RNA polymerase II transcription, and were previously thought to have no biological function (16). IncRNAs do not encode proteins, but play a critical role in gene regulation and epigenetic modifications (17-19). IncRNA affect cartilage matrix production and metabolism, synovitis, autophagy and apoptosis of chondrocytes $(20,21)$. Gaballah et al (18) reported that ZNF1 antisense RNA 1 can promote chondrocyte proliferation and inhibit apoptosis by regulating the expression level of Wnt family member 3A. A previous study by Wang et al (22) suggested that nuclear paraspeckle assembly transcript 1 can act on microRNA-181c to promote the proliferation of human synoviocytes and accelerate the progression of osteoarthritis (22). Although the specific function of lncRNAs has not been elucidated, previous studies have reported that the abnormal expression of lncRNAs is closely related to osteoarthritis $(23,24)$.

The aim of the present study was to examine the lncRNA expression profiles of cartilage from young patients with amputations without joint lesions, and from patients with knee osteoarthritis who underwent total knee arthroplasty. The lncRNA expression profiles of 13 patients were compared to identify new potential regulatory targets involved in the pathogenesis of the disease. The results of the present study may aid in identifying the role of lncRNAs in the progression of knee osteoarthritis.

\section{Materials and methods}

Subjects. The present study recruited 13 patients from the Department of Orthopedic Surgery, The Second Affiliated Hospital of Anhui Medical University, between February 2016 and December 2018, including six males and seven females. The 13 patients were divided into two groups: Six patients aged 29-49 years in the normal group (NC), and seven patients aged 67-82 years in the knee osteoarthritis group (OA; average age $38.17 \pm 7.70$ vs. $71.71 \pm 6.02$ years). The NC group was made up of young patients with amputations without joint disease. Inclusion criteria for the NC group were as follows: i) No history of joint lesions; and ii) lower limb injury treated with amputation. All patients in the OA group were patients with knee osteoarthritis who underwent total knee arthroplasty. The inclusion criteria for the OA group were as follows: i) Diagnosed with knee osteoarthritis based on clinical manifestations, combined with X-ray examination, knee pain and severely affected quality of life, with failure of conventional oral drug therapy; ii) patients with primary total knee arthroplasty; iii) knee flexion malformation $<15^{\circ}$, varus deformity $<15^{\circ}$ or valgus deformity $<15^{\circ}$; and iv) Kellgren Lawrence III-IV level (25). Exclusion criteria for both groups were as follows: i) Severe internal and external valgus and flexion contracture deformity (flaw angle $>15^{\circ}$ ); ii) obese patients (body mass index $>35$ ); iii) patients with heart and lung dysfunction, cerebrovascular disease or diabetes; iv) history of open joint surgery; and v) active infection. The present study was approved by The Second Affiliated Hospital of Anhui Medical University
Ethics Committee. Written informed consent was obtained from each patient or their relatives prior to surgery.

Sample collection and total RNA extraction. Approximately $5 \mathrm{~g}$ of cartilage tissue was collected from each patient and stored at $-80^{\circ} \mathrm{C}$ until further analysis. Total RNA was extracted from the tissue suspension using the TRIzol ${ }^{\circledR}$ reagent (Invitrogen; Thermo Fisher Scientific, Inc.) according to the manufacturer's instructions. The tissue was homogenized prior to RNA extraction. The amount and purity of the total RNA were assessed using a NanoDrop 2000 spectrophotometer (Thermo Fisher Scientific, Inc.), and RNA integrity was analyzed using an RNA Nano 6000 kit from the Agilent Bioanalyzer 2100 system (Agilent Technologies, Inc.).

Preparation for lncRNA-sequencing library. A lncRNA library was constructed using $3 \mu \mathrm{g}$ of total RNA per sample. The Ribo-Zero ${ }^{\mathrm{TM}}$ Gold kit (Illumina, Inc.) was used to remove the rRNA from the sample and select the different index tags to build the library according to the manufacturer's instructions. The NEB Next Ultra ${ }^{\mathrm{TM}}$ Directional RNA Library Prep kit for Illumina (New England BioLabs, Inc.) was used to construct a sequencing library using rRNA-depleted RNA, according to the manufacturer's instructions.

High-throughput sequencing. Sequencing libraries were generated using the NEBNext Ultra ${ }^{\mathrm{TM}}$ Directional RNA LibraryPrep kit for Illumina (New England BioLabs, Inc.) according to the manufacturer's protocol. Raw reads were obtained by Illumina sequencing and high-quality sequences (clean reads) were obtained through a series of data processing procedures such as removing low-quality sequences, de-linking contamination and rRNA including removing reads of joint contamination (the base number of joint contamination in reads was greater than 5 bp, removing low-quality reads (reads intermediate value $\mathrm{Q} \leq 19$ accounted for $>50 \%$ of the total base), removing reads with $\mathrm{N}$ ratio $>5 \%$ and removing reads matched with rRNA.

All subsequent analyses were based on clean reads. Library sequencing was carried out on a HiSeq 4000 platform (Illumina, Inc.) according to the commercially available protocols from Chongqing Western Biotechnology, Inc.

Identification of differentially expressed lncRNAs. The analysis of differences in IncRNA expression between the two groups (NC and OA group) was performed using the DEGseq (2010) R package (http://dblp.uni-trier.de/db/journals/bioinformatics/bioinformatics26.html\#WangFWWZ10). The $\mathrm{P}$-value was adjusted using the q-value. $\mathrm{P}<0.05$ and $\mid \log 2$ (fold-change) $\mid>1$ were set as the threshold for significant differential expression.

Reverse transcription-quantitative PCR (RT-qPCR). To validate the reliability of the RNA-sequencing data, 6 differentially expressed lncRNAs were randomly selected and RT-qPCR was used to examine the expression level of the lncRNAs. Total RNA was extracted from the cartilage using the TRIzol reagent (Invitrogen, Thermo Fisher 
Scientific, Inc.) according to the manufacturer's instructions. RT-qPCR reactions were performed using the Luna Universal One-Step RT-qPCR kit (New England BioLabs, Inc.) according to the manufacturer's instructions. The following thermocycling conditions were used: $15 \mathrm{sec}$ at $55^{\circ} \mathrm{C}$ and $1 \mathrm{~min}$ at $95^{\circ} \mathrm{C}$, followed by 40 cycles of $10 \mathrm{sec}$ at $95^{\circ} \mathrm{C}$ and $30 \mathrm{sec}$ at $60^{\circ} \mathrm{C}$, and $30 \mathrm{sec}$ at $50^{\circ} \mathrm{C}$. GAPDH was used as an internal control and the relative expression levels of candidate lncRNAs were calculated using the $2^{-\Delta \Delta \mathrm{Cq}}$ method (26). GAPDH forwards primer, AATGGG CAGCCGTTAGGAAA and reverse primer, GCCCAATAC GACCAAATCAGAG. Experiments were conducted in at least triplicate. The primer sequences that were used in the present study are listed in Table SI.

GO and KEGG enrichment analyses. Target mRNAs of the differentially expressed lncRNAs were classified according to the principles of GO classification. GO collects information from GO and NCBI databases, annotating and classifying genes based on biological processes, molecular functions and cellular components (http://www.geneontology.org/). KEGGis a comprehensive database for the systematic analysis of gene function, which is based on manually drawn metabolic pathways and is divided into metabolism, genetic information processing, cellular processes, environmental information processing, biological systems and human diseases (http://www.genome.jp/kegg/).

Statistical analysis. Data analyses were performed using SPSS software (version 19.0; IBM Corp.) and data are expressed as the mean \pm SD. Comparisons of data between groups were performed by the Student's t-test. Categorical data were analyzed by the $\chi^{2}$ test. $\mathrm{P}<0.05$ was considered to indicate a statistically significant difference.

\section{Results}

Clinical characteristics. The clinical characteristics of the 13 patients are presented in Table I. The NC group consisted of six patients including three men and three women with a mean age of $38.17 \pm 7.70$ years. The OA group consisted of seven patients including three women and three men with a mean age of $71.71 \pm 6.02$. A significant difference was identified between the ages of the two groups $(\mathrm{P}=0.001)$. There were no significant differences between the two groups regarding sex, $\mathrm{BMI}$ and limb side $(\mathrm{P}=0.817,0.389$ and 0.751 , respectively).

Differentially expressed lncRNAs. A total of 23,583 lncRNAs were identified in osteoarthritis cartilage, including 5,255 upregulated and 5,690 downregulated lncRNAs, compared with normal cartilage ( $\log 2$ fold-change $>1 ; \mathrm{P}<0.05$; Fig. 1 ). The most significantly upregulated lncRNA was MSTRG.95856 ( $\log 2$ fold-change $=11.6488$ ). The most significantly downregulated lncRNA was ENSG00000279725 (log2 fold-change = -9.6546). According to the microarray data, the number of downregulated lncRNAs was larger than that of the upregulated lncRNAs. However, among significantly differentially expressed lncRNAs ( $\log 2$ fold-change $>6$ ), there were more upregulated than downregulated lncRNAs. Hierarchical clustering analysis displayed
Table I. Baseline clinical characteristics.

\begin{tabular}{lccc}
\hline Variable & $\mathrm{NC}, \mathrm{n}=6$ & $\mathrm{OA}, \mathrm{n}=7$ & $\mathrm{P}$-value \\
\hline Age, years (mean $\pm \mathrm{SD})$ & $38.17 \pm 7.70$ & $71.71 \pm 6.02$ & 0.001 \\
Sex (male/female) & $3 / 3$ & $3 / 4$ & 0.817 \\
BMI (mean $\pm \mathrm{SD})$ & $20.82 \pm 2.11$ & $22.16 \pm 3.09$ & 0.389 \\
Side (left/right) & $2 / 4$ & $4 / 3$ & 0.751 \\
\hline
\end{tabular}

$\mathrm{NC}$, normal control group; OA, osteoarthritis group; BMI, body mass index.

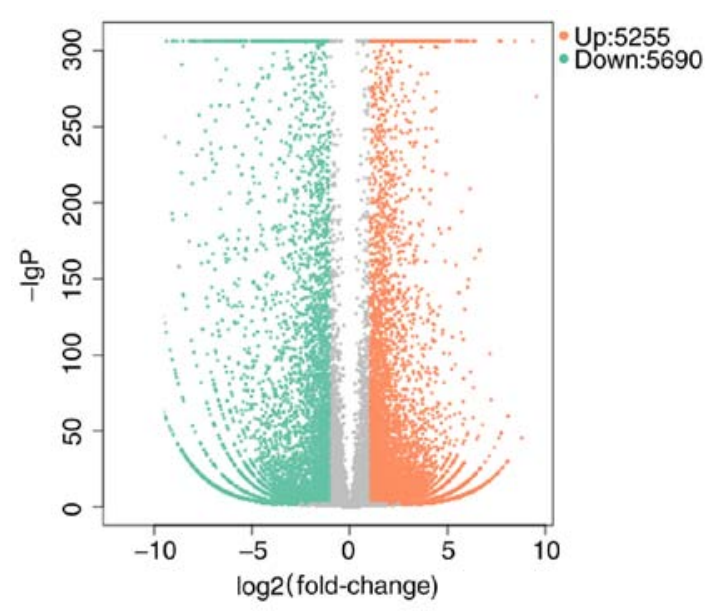

Figure 1. Volcano plot of differential lncRNA expression (OA vs. NC group). $\mathrm{X}$-axis, $\log 2$ (fold-change). Y-axis, $-1 \mathrm{x} \log 10$ (corrected P-value) for each probe. Grey dots represent no differential lncRNA expression. IncRNA, long non-coding RNA; OA, osteoarthritis; NC, normal control; up, upregulated; down, downregulated.

the differential expression of IncRNAs (Fig. 2), where red and blue represent expression values higher and lower than the median expression value, respectively. The results indicated that differences between osteoarthritis and normal cartilage could be identified by differences in the expression profiles of lncRNAs associated with osteoarthritis (Table II).

GO and KEGG pathway analyses. IncRNAs do not encode proteins and they function through cis- or trans-regulation of protein-coding genes (27). For the target genes of differentially expressed lncRNAs, the functions of these lncRNAs were indirectly predicted according to the target genes. GO and KEGG pathway analyses of the target genes of the differential lncRNAs were then performed.

Table III and Fig. 3 display the top 10 enriched GO entries with the most significant difference in target genes of lncRNAs in each group. In biological processes, target genes were mainly enriched in the metabolic process of substances, including 'organic substance metabolic process'. In cellular components, target genes were mainly enriched in 'intracellular part'. In molecular functions, target genes were mainly enriched in 'binding'. However, target genes do not appear to be mainly enriched in all of the terms listed here. Furthermore, Table IV and Fig. 4 display the KEGG pathways with the most significant 


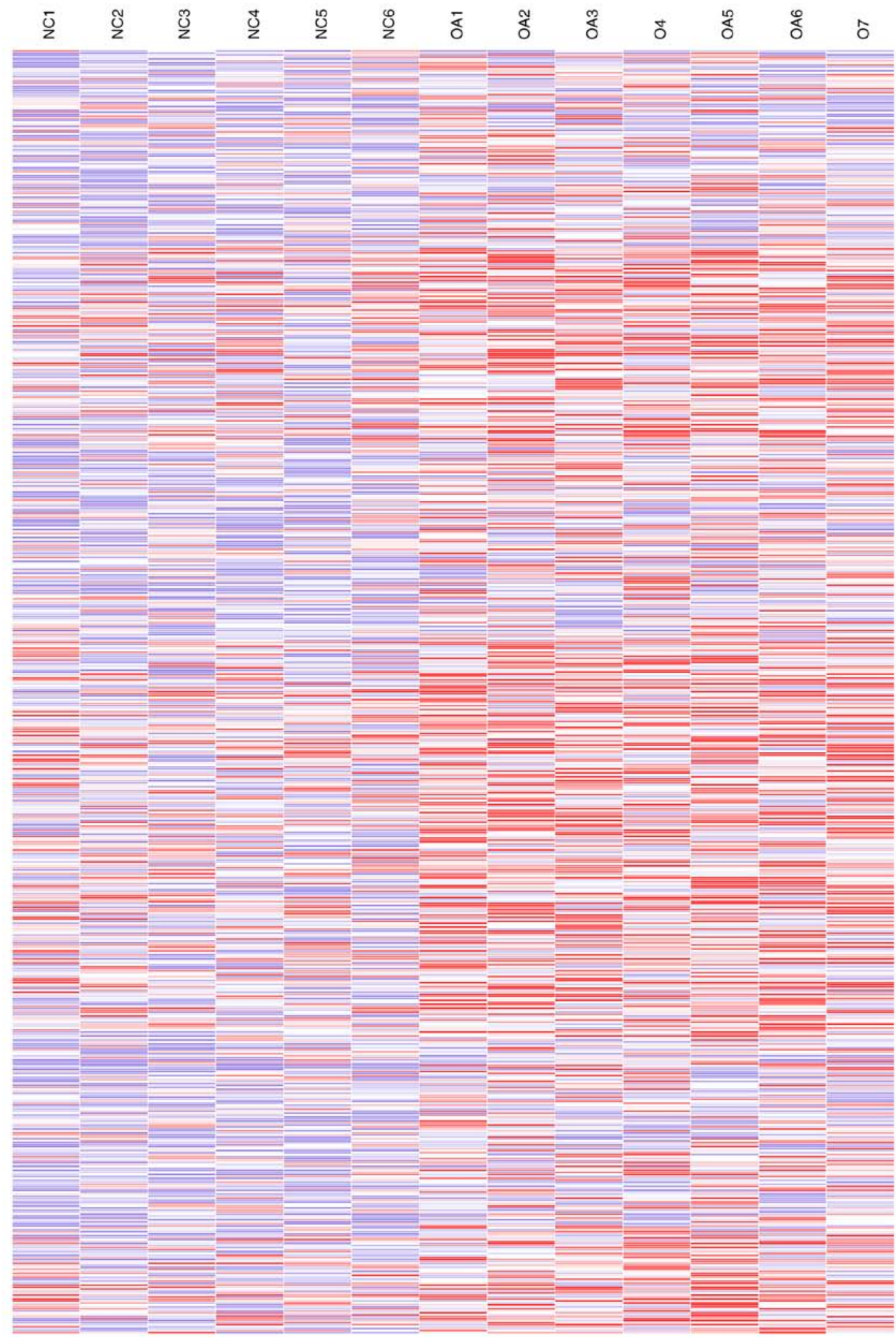

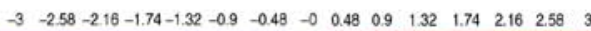

Figure 2. Hierarchical clustering of lncRNAs in the NC and OA groups. The red and blue shaded areas indicate increased and decreased expression levels, respectively, across all samples. The white areas indicate no differential lncRNA expression. IncRNA, long non-coding RNA; OA, osteoarthritis; NC, normal control.

differences in target genes for IncRNAs between the two groups. In the KEGG pathway analysis, the enrichment pathways associated with significant expression of lncRNAs were 'PI3K-Akt signaling pathway', 'endocytosis', 
Table II. Most significantly expressed top 15 entries in upregulated and downregulated lncRNAs.

\begin{tabular}{|c|c|c|c|c|}
\hline lncRNA ID & Position & Log2 (fold-change) & Regulation & P-value \\
\hline MSTRG.95856 & chr17:64319415-64413776 & 11.64880695 & Up & $8.83 \times 10^{-245}$ \\
\hline ENSG00000246430 & chr8:56436674-56446734 & 11.12541347 & Up & $3.69 \times 10^{-122}$ \\
\hline ENSG00000253730 & chr17:50183289-50201632 & 9.970804581 & Up & $4.75 \times 10^{-116}$ \\
\hline ENSG00000261959 & $\operatorname{chr17:50183289-50201632}$ & 9.970804581 & Up & $4.35 \times 10^{-241}$ \\
\hline ENSG00000214145 & chr3:194355247-194369743 & 9.961630563 & Up & $2.60 \times 10^{-221}$ \\
\hline ENSG00000254855 & chr11:66314487-66317044 & 9.259743264 & Up & $4.07 \times 10^{-232}$ \\
\hline ENSG00000255468 & chr11:66314487-66317044 & 9.259743264 & $\mathrm{Up}$ & $2.53 \times 10^{-206}$ \\
\hline ENSG00000269053 & chr19:17351448-17377350 & 8.885696373 & $\mathrm{Up}$ & $2.93 \times 10^{-190}$ \\
\hline ENSG00000282851 & chr19:17351448-17377350 & 8.885696373 & Up & $7.99 \times 10^{-111}$ \\
\hline MSTRG.106332 & chr19:17351448-17377350 & 8.885696373 & $\mathrm{Up}$ & $3.09 \times 10^{-214}$ \\
\hline MSTRG.143488 & chr22:39223359-39244751 & 8.774787060 & Up & $4.01 \times 10^{-172}$ \\
\hline MSTRG.92410 & chr17:31303766-31314112 & 8.727920455 & Up & $6.30 \times 10^{-233}$ \\
\hline ENSG00000227502 & chr6:113857362-113863471 & 8.715226609 & Up & $3.50 \times 10^{-94}$ \\
\hline ENSG00000244161 & chr3:58192257-58214697 & 8.640244936 & Up & $7.63 \times 10^{-237}$ \\
\hline ENSG00000258810 & chr14:20801228-20803278 & 8.628141719 & Up & $5.65 \times 10^{-160}$ \\
\hline ENSG00000279725 & chr10:127737235-127741186 & -9.654636029 & Down & $1.26 \times 10^{-23}$ \\
\hline ENSG00000280302 & chr18:12254319-12277595 & -8.214319121 & Down & $1.22 \times 10^{-79}$ \\
\hline ENSG00000250012 & chr3:126103562-126197994 & -7.740118043 & Down & $6.01 \times 10^{-116}$ \\
\hline MSTRG.155259 & chr3:126103562-126197994 & -7.740118043 & Down & $7.25 \times 10^{-14}$ \\
\hline ENSG00000231966 & chr1:179743163-179816198 & -6.693486957 & Down & $1.35 \times 10^{-23}$ \\
\hline ENSG00000203288 & $\operatorname{chr} 1: 151806071-151831872$ & -6.524023102 & Down & $4.52 \times 10^{-102}$ \\
\hline ENSG00000234614 & chr1:151806071-151831872 & -6.524023102 & Down & $3.04 \times 10^{-170}$ \\
\hline MSTRG.13443 & chr1:151806071-151831872 & -6.524023102 & Down & $8.04 \times 10^{-48}$ \\
\hline ENSG00000229178 & chr3:195614947-195620233 & -6.438791853 & Down & $6.39 \times 10^{-61}$ \\
\hline ENSG00000242086 & chr3:195614947-195620233 & -6.438791853 & Down & $8.84 \times 10^{-211}$ \\
\hline MSTRG.162045 & chr3:195614947-195620233 & -6.438791853 & Down & $4.62 \times 10^{-19}$ \\
\hline MSTRG.162046 & chr3:195614947-195620233 & -6.438791853 & Down & $5.26 \times 10^{-151}$ \\
\hline ENSG00000227388 & chr9:35752990-35756613 & -6.384897330 & Down & $3.85 \times 10^{-29}$ \\
\hline ENSG00000226133 & chr1:48102068-48104707 & -6.266786541 & Down & $2.40 \times 10^{-70}$ \\
\hline ENSG00000261026 & chr8:22713251-23000000 & -6.200762405 & Down & $1.36 \times 10^{-271}$ \\
\hline
\end{tabular}

lncRNA, long non-coding RNA.

'cytokine-cytokine receptor interaction' and 'chemokine signaling pathway'.

$R T-q P C R$ validation of IncRNA expression. To verify the sequencing results of the IncRNAs, three upregulated lncRNAs (ENSG00000261496, ENSG00000273447 and ENSG00000269971) and three downregulated lncRNAs (ENSG00000257477, ENSG00000254369 and MSTRG.119041) were randomly selected. The expression levels of the six lncRNAs were detected by RT-qPCR in 13 cartilage samples (six NC and seven OA). The results of the RT-qPCR analysis were consistent with the microarray data (Fig. 5). Each lncRNA displayed a similar trend of upregulation or downregulation.

\section{Discussion}

IncRNAs do not encode proteins, but function at the RNA level, regulating gene expression and epigenetic modifications (28). Abnormal expression of lncRNAs is involved in the pathogenesis of a number of diseases, such as glioma, allergic dermatitis and rheumatism (29-31). Previous studies have reported that the abnormal expression of lncRNAs in osteoarthritis cartilage in vitro is related to the degradation of the extracellular matrix of chondrocytes, suggesting that lncRNAs are involved in the pathogenesis of osteoarthritis $(32,33)$. However, the potential targets and functions of lncRNAs associated with skeletal development and osteoarthritis are not fully understood. The present study systematically screened the lncRNA expression profiles of cartilage from normal control patients and patients with knee osteoarthritis to identify novel lncRNA targets involved in the mechanisms underlying osteoarthritis. The new lncRNA targets might aid in identifying the role of lncRNAs in the progression of knee osteoarthritis.

The results displayed differences in the expression levels of a number of lncRNAs between normal and osteoarthritis 
Table III. Top 15 significantly enriched GO terms in biological processes, cellular components and molecular functions of the differentially expressed lncRNAs.

A, Upregulated

\begin{tabular}{lllll}
\hline Category & GO accession no. & \multicolumn{1}{c}{ Description } & Corrected P-value & Total number of genes \\
\hline GOTERM_BP & GO:0008152 & Metabolic process & $7.1 \times 10^{-11}$ & 4,779 \\
GOTERM_BP & GO:0071704 & Organic substance metabolic process & $4.2 \times 10^{-11}$ & 4,573 \\
GOTERM_BP & GO:0044237 & Cellular metabolic process & $2.7 \times 10^{-14}$ & 4,486 \\
GOTERM_BP & GO:0009058 & Biosynthetic process & $2.6 \times 10^{-09}$ & 2,858 \\
GOTERM_BP & GO:1901576 & Organic substance biosynthetic process & $2.7 \times 10^{-09}$ & 2,820 \\
GOTERM_CC & GO:0043226 & Organelle & $2.5 \times 10^{-17}$ & 5,787 \\
GOTERM_CC & GO:0043229 & Intracellular organelle & $6.0 \times 10^{-20}$ & 5,414 \\
GOTERM_CC & GO:0043227 & Membrane-bounded organelle & $1.4 \times 10^{-18}$ & 5,316 \\
GOTERM_CC & GO:0043231 & Intracellular membrane-bounded organelle & $3.9 \times 10^{-22}$ & 4,902 \\
GOTERM_CC & GO:0005737 & Cytoplasm & $2.2 \times 10^{-11}$ & 4,784 \\
GOTERM_MF & GO:0097159 & Organic cyclic compound binding & $2.9 \times 10^{-6}$ & 2,861 \\
GOTERM_MF & GO:1901363 & Heterocyclic compound binding & $2.3 \times 10^{-6}$ & 2,836 \\
GOTERM_MF & GO:0043167 & Ion binding & $6.6 \times 10^{-5}$ & 2,639 \\
GOTERM_MF & GO:0043169 & Cation binding & $2.5 \times 10^{-5}$ & 1,804 \\
GOTERM_MF & GO:0046872 & Metal ion binding & 1,779 \\
\hline
\end{tabular}

B, Downregulated

\begin{tabular}{llllc}
\hline Category & GO accession no. & \multicolumn{1}{c}{ Description } & Corrected P-value & Total number of genes \\
\hline GOTERM_BP & GO:0044238 & Primary metabolic process & $6.6 \times 10^{-11}$ & 4,424 \\
GOTERM_BP & GO:0043170 & Macromolecule metabolic process & $7.5 \times 10^{-15}$ & 3,935 \\
GOTERM_BP & GO:0044260 & Cellular macromolecule metabolic process & $4.4 \times 10^{-17}$ & 3,660 \\
GOTERM_BP & GO:0006807 & Nitrogen compound metabolic process & $7.8 \times 10^{-09}$ & 2,947 \\
GOTERM_BP & GO:0044249 & Cellular biosynthetic process & $1.6 \times 10^{-10}$ & 2,781 \\
GOTERM_CC & GO:0005622 & Intracellular & $1.0 \times 10^{-29}$ & 6,263 \\
GOTERM_CC & GO:0044424 & Intracellular part & $3.0 \times 10^{-27}$ & 6,127 \\
GOTERM_CC & GO:0044422 & Organelle part & $1.6 \times 10^{-7}$ & 3,740 \\
GOTERM_CC & GO:0044446 & Intracellular organelle part & $8.1 \times 10^{-8}$ & 3,675 \\
GOTERM_CC & GO:0044444 & Cytoplasmic part & $4.1 \times 10^{-5}$ & 3,572 \\
GOTERM_MF & GO:0005488 & Binding & $2.6 \times 10^{-6}$ & 4,991 \\
GOTERM_MF & GO:0003824 & Catalytic activity & $5.0 \times 10^{-5}$ & $1.9 \times 10^{-4}$ \\
GOTERM_MF & GO:0003676 & Nucleic acid binding & $8.9 \times 10^{-10}$ & 1,994 \\
GOTERM_MF & GO:0003677 & DNA binding & $1.1 \times 10^{-5}$ & 1,185 \\
GOTERM_MF & GO:0003700 & Transcription factor activity, & 526 \\
\hline
\end{tabular}

GO, gene ontology; lncRNA, long non-coding RNA.

cartilage, ranging from a few to several thousands. The variable differences may be attributed to the small sample size. However, RT-qPCR was performed to verify the functions of randomly selected lncRNAs. The results suggested that the trend was similar to that of the microarray data, which provided evidence of differential lncRNA expression levels between normal and osteoarthritis cartilage.

Bioinformatics methods were used to perform cis- and trans-target analyses to identify the target genes of differentially expressed lncRNAs, as it is difficult to predict the function of lncRNA based solely on nucleotide sequences $(34,35)$. Then, the target genes were subjected to GO and KEGG enrichment analyses to explore the regulation of lncRNAs according to target gene function.

Target analysis identified a number of potential lncRNA regulatory targets. Certain targets were previously reported to play an important role in the pathogenesis of osteoarthritis, although their potential function as regulatory targets for lncRNA shad not previously been suggested $(36,37)$. For 


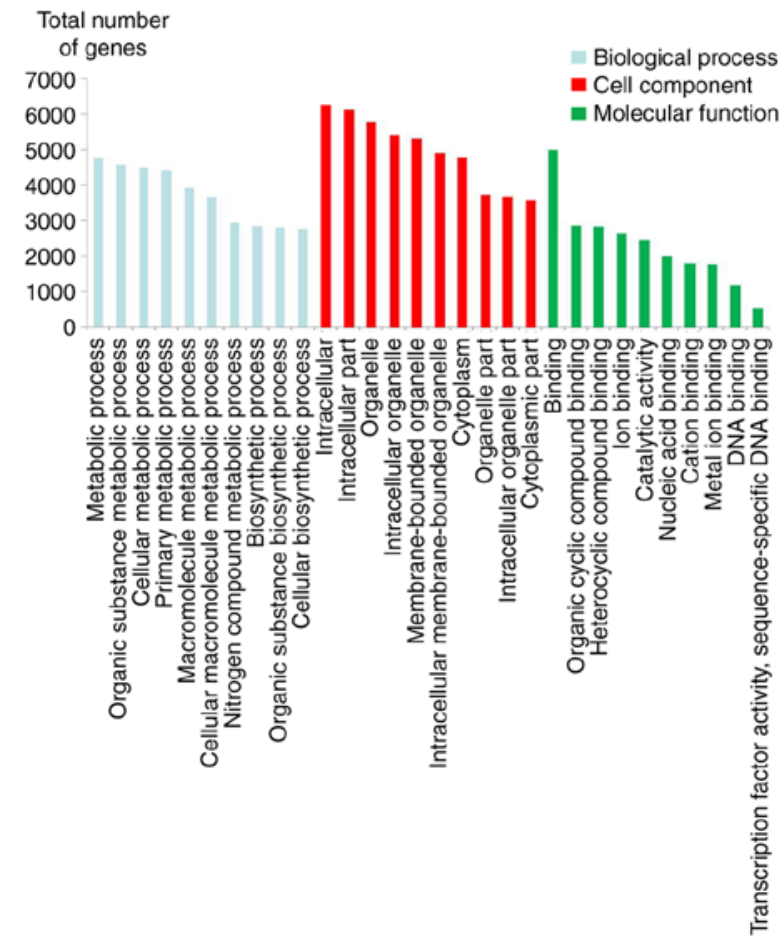

Figure 3. GO analysis of differentially expressed lncRNAs covering three domains: Biological processes, cellular components and molecular functions. The red columns indicate terms associated with cell components, the green columns indicate terms associated with molecular functions and the blue columns indicate terms associated with biological processes. GO, Gene Ontology; lncRNA, long non-coding RNA.

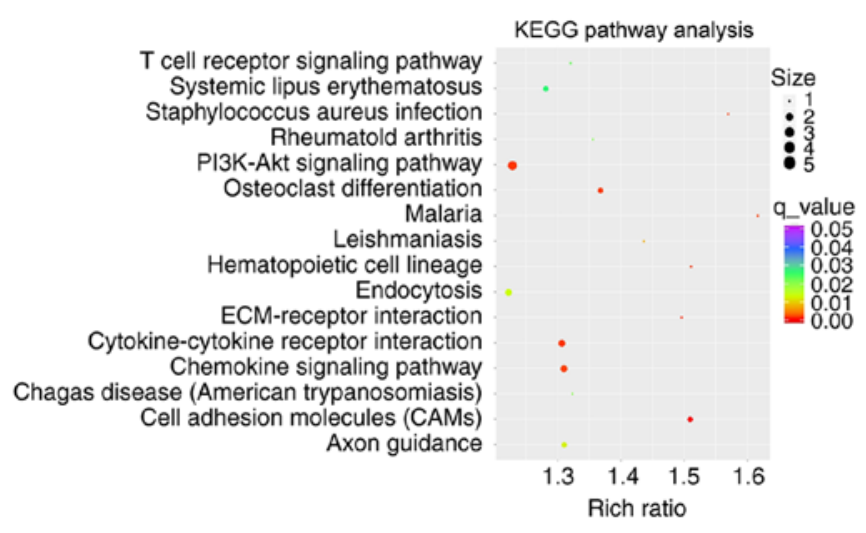

Figure 4. KEGG pathway analysis of differentially expressed lncRNAs. Each dot represents the degree of enrichment of the KEGG entry. The closer the color is to red, the higher the enrichment. The size of each dot represents the number of genes enriched in the KEGG entry; the larger the dot, the more genes are enriched in the KEGG entry. Rich ratio: (Differential genes of this pathway/all differential genes)/(genes annotated to this pathway/all can be annotated to Gene). KEGG, Kyoto Encyclopedia of Genes and Genomes; lncRNA, long non-coding RNA.

example, in the present study, integrin- $\alpha 5$ (ITGA5) was found to be a potential target of IncRNA ENSG00000257477, which is expressed at a 2.2787-fold higher level in normal cartilage than in osteoarthritis chondrocytes. ITGA5 can mediate mutual adhesion and two-way signal transduction between chondrocytes and the extracellular matrix (38). ITGA5 is also involved in physiological processes including inflammation, differentiation and migration (39), and the

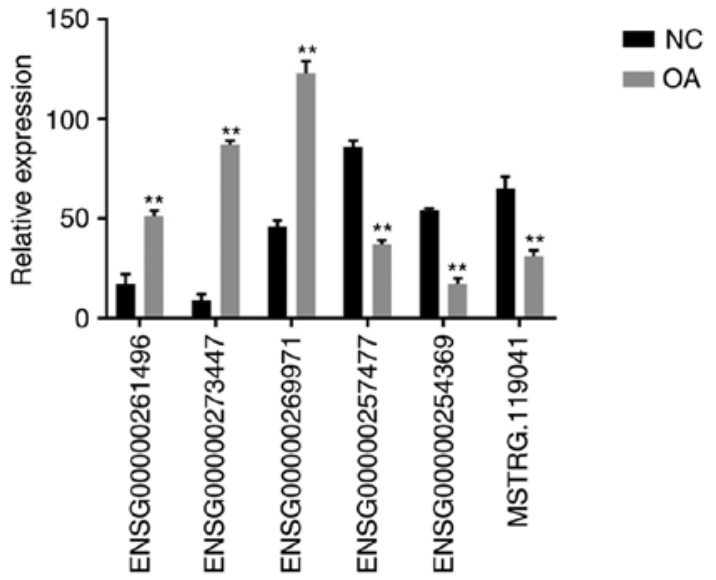

Figure 5. Validation of RNA-sequencing results by RT-qPCR. The data are expressed as the mean $\pm \mathrm{SD} .{ }^{* *} \mathrm{P}<0.01$ vs. NC. RT-qPCR, reverse transcription-quantitative PCR; OA, osteoarthritis group; NC, normal control group.

dedifferentiation of chondrocytes leads to a reduction in ITGA5 (40). Another example is JNK, a potential target of lncRNA ENSG00000261496, which was found in the present study to be expressed at a 4.4304-fold higher level in osteoarthritis than in normal chondrocytes. Elevated levels of JNK and activation of the JNK signaling pathway may aggravate chondrocyte inflammation and cause cartilage degradation (41). Mice overexpressing JNK are more susceptible to osteoarthritis compared to normal mice (42). In the present study, certain targets that have not previously been reported to play a role in osteoarthritis were identified as having a potential regulatory role in osteoarthritis. For example, high mobility group protein B1 (HMGB1), a late inflammatory regulator, has been studied for its role in coronary atherosclerosis and diabetes $(43,44)$. However, results from the present study suggested that the expression level of HMGB1 was higher in articular cartilage tissue than in normal cartilage ( $\sim 22.0320$-fold), indicating that it may be a regulatory target of IncRNA ENSG00000272825, which plays a proinflammatory role (45). Interferon induced transmembrane protein 3 (IFITM3), a member of the IFITM gene family, is involved in cell adhesion, immune cell regulation and stem cell differentiation and maturation (46). IFITM3also plays a role in tumor proliferation and viral infection (47). Microarray data suggested that IFITM3 may be the regulatory target of lncRNA ENSG00000277310. The expression level of IFITM3 was lower in arthritic cartilage than in normal cartilage, which may be related to the continuous dedifferentiation of chondrocytes [(48), (Fig S1)].

The PI3K-Akt signaling pathway is involved in the release of the proinflammatory cytokines IL- $1 \alpha$, IL-1 $\beta$, TNF- $\alpha$ and IL- 6 and the regulation of the joint inflammatory microenvironment, based on significantly abundant KEGG signaling pathways. The present study suggested that the PI3K-Akt signaling pathway is related to the occurrence and progression of knee osteoarthritis. In addition, the present study identified several chemokine signaling pathways that are involved in the differentiation and metabolism of osteoclasts, which may also be related to the development of joint inflammation (49). 
Table IV. KEGG pathway analysis of the differentially expressed lncRNAs.

A, Upregulated

\begin{tabular}{lllr}
\hline KEGG pathway & \multicolumn{1}{c}{ Term } & P-value & Total number of genes \\
\hline hsa04320 & PI3K-Akt signaling pathway & $1.4458 \times 10^{-5}$ & 214 \\
hsa05630 & Hematopoietic cell lineage & $6.3426 \times 10^{-6}$ & 60 \\
hsa04722 & Osteoclast differentiation & $2.0712 \times 10^{-5}$ & 92 \\
hsa05803 & Staphylococcus aureus infection & $2.3805 \times 10^{-5}$ & 44 \\
hsa03638 & Chemokine signaling pathway & $3.8041 \times 10^{-5}$ & 115 \\
hsa04327 & Malaria & $3.6349 \times 10^{-5}$ & 37 \\
hsa02874 & Leishmaniasis & $2.4737 \times 10^{-4}$ & 51 \\
hsa00381 & Axon guidance & $4.6875 \times 10^{-4}$ & 82 \\
\hline
\end{tabular}

B, Downregulated

\begin{tabular}{llrr}
\hline KEGG pathway & \multicolumn{1}{c}{ Term } & P-value & Total number of genes \\
\hline hsa00273 & Cell adhesion molecules (CAMs) & $8.5728 \times 10^{-09}$ & 98 \\
hsa03256 & Cytokine-cytokine receptor interaction & $1.416 \times 10^{-5}$ & 130 \\
hsa04438 & ECM-receptor interaction & $1.484 \times 10^{-5}$ & 58 \\
hsa02931 & Endocytosis & $5.5678 \times 10^{-4}$ & 141 \\
hsa01375 & Chagas disease (American trypanosomias) & $9.3938 \times 10^{-4}$ & 68 \\
hsa02306 & Rheumatoid arthritis & $1.03056 \times 10^{-3}$ & 57 \\
hsa00561 & T cell receptor signaling pathway & $1.20597 \times 10^{-3}$ & 66 \\
hsa01934 & Systemic lupus erythematosus & $1.49979 \times 10^{-3}$ & 79 \\
\hline
\end{tabular}

KEGG, Kyoto Encyclopedia of Genes and Genomes; lncRNA, long non-coding RNA.

The present study had several limitations. Firstly, the age of the patients in the $\mathrm{NC}$ group was lower than that of the patients in the OA group, therefore, a number of the detected transcripts may be age-related rather than disease-specific. Future studies should include a larger number of age-matched control cartilage samples. Secondly, as only a few patients agreed to donate knee cartilage, the present study consisted of a small sample size. In order to provide support for the conclusions made in the present study, further investigations should include larger cohorts. Thirdly, only indirect experimental evidence of the functional link between lncRNAs and their predicted target genes was included in the present study. Therefore, this also requires further investigation.

In summary, the present study used RNA microarray data to describe the expression profiles of lncRNAs in osteoarthritis and normal cartilage. Bioinformatics methods were used to predict the target genes and potential functions of differentially expressed lncRNAs. The results obtained by microarray analysis may contribute to the diagnosis of osteoarthritis and predict the development of osteoarthritis. Differentially expressed lncRNAs may also help to identify novel potential candidate targets for the treatment of knee osteoarthritis.

\section{Acknowledgements}

Not applicable.

\section{Funding}

The present study received funding from the Major Research and Development Project of Anhui Department of Science and Technology (grant no. 1804h08020269).

\section{Availability of data and materials}

The datasets used and/or analyzed in the current study are available from the corresponding author on reasonable request.

\section{Authors' contributions}

YL and WC analyzed and interpreted the sequencing data regarding osteoarthritis and contributed to writing the manuscript. JJ designed the study. HY, JZ and QC performed the surgeries and collected tissue. XZ, JL and SZ performed secondary analysis and production of partial sequencing data and charts. All authors read and approved the final manuscript.

\section{Ethics approval and consent to participate}

The present study was approved by The Second Affiliated Hospital of Anhui Medical University Ethics Committee (approval no. SL-YX2019-019). Written informed consent was obtained from each patient or their relatives prior to surgery. 


\section{Patient consent for publication}

Not applicable.

\section{Competing interests}

The authors declare that they have no competing interests.

\section{References}

1. Health Quality Ontario: Structured education and neuromuscular exercise program for Hip and/or knee osteoarthritis: A health technology assessment. Ont Health Technol Assess Ser 18: 1-110, 2018.

2. Zheng S, Tu L, Cicuttini F, Han W, Zhu Z, Antony B, Wluka A, Winzenberg T, Meng T, Aitken D, et al: Effect of Vitamin D supplementation on depressive symptoms in patients with knee osteoarthritis. J Am Med Dir Assoc 20: 1634-1640.e1, 2019.

3. Musumeci G, Loreto C, Imbesi R, Trovato FM, Di Giunta A, Lombardo C, Castorina S and Castrogiovanni P: Advantages of exercise in rehabilitation, treatment and prevention of altered morphological features in knee osteoarthritis. A narrative review. Histol Histopathol 29: 707-719, 2014.

4. Petersen KK, Olesen AE, Simonsen O and Arendt-Nielsen L: Mechanistic pain profiling as a tool to predict the efficacy of 3 -weeks non-steroidal anti-inflammatory drugs (NSAIDs) plus paracetamol in patients with painful knee osteoarthritis. Pain 160: 486-492, 2019

5. Liang Y, Idrees E, Szojka A, Andrews S, Kunze M, Mulet-Sierra A, Jomha $\mathrm{N}$ and Adesida AB: Chondrogenic differentiation of synovial fluid mesenchymal stem cells on human meniscus-derived decellularized matrix requires exogenous growth factors. Acta Biomater 80: 131-143, 2018.

6. Driban JB, Eaton CB, Amin M, Stout AC, Price LL, Lu B, Lo GH, McAlindon TE and Barbe MF: Glucose homeostasis influences the risk of incident knee osteoarthritis: Data from the osteoarthritis initiative. J Orthop Res 35: 2282-2287, 2017.

7. Thorson C, Galicia K, Burleson A, Bouchard O, Hoppensteadt D, Fareed $\mathbf{J}$ and Hopkinson $\mathrm{W}$ : Matrix metalloproteinases and their inhibitors and proteoglycan 4 in patients undergoing total joint arthroplasty. Clin Appl Thromb Hemost 25: 1076029619828113 , 2019.

8. Davis HC, Luc-Harkey BA, Seeley MK, Troy Blackburn J and Pietrosimone B: Sagittal plane walking biomechanics in individuals with knee osteoarthritis after quadriceps strengthening. Osteoarthritis Cartilage 27: 717-780, 2019.

9. Zhou X, Jiang L, Zhang Y, Zhang J, Zhou D, Wu L, Huang Y and Xu N: Genetic variation of aggrecanase-2 (ADAMTS5) in susceptibility to osteoarthritis. Braz J Med Biol Res 52: e8109, 2019.

10. Bar-Or D, Thomas G, Rael LT, Frederick E, Hausburg M, Bar-Or R and Brody E: On the mechanisms of action of the low molecular weight fraction of commercial human serum albumin in osteoarthritis. Curr Rheumatol Rev 15: 189-200, 2019.

11. Giunta S, Castorina A, Marzagalli R, Szychlinska MA, Pichler K, Mobasheri A and Musumeci G: Ameliorative effects of PACAP against cartilage degeneration. morphological, immunohistochemical and biochemical evidence from in vivo and in vitro models of rat osteoarthritis. Int J Mol Sci 16: 5922-5944, 2015.

12. Gauffin H, Sonesson S, Meunier A, Magnusson H and Kvist J: Knee arthroscopic surgery in middle-aged patients with meniscal symptoms: A 3-year follow-up of a prospective, randomized study. Am J Sports Med 45: 2077-2084, 2017.

13. Tasci Bozbas G, Sendur OF and Aydemir AH: Primary knee osteoarthritis increases the risk of falling. J Back Musculoskelet Rehabil 30: 785-789, 2017.

14. Castrogiovanni P, Di Rosa M, Ravalli S, Castorina A, Guglielmino C, Imbesi R, Vecchio M, Drago F, Szychlinska MA and Musumeci G: Moderate physical activity as a prevention method for knee osteoarthritis and the role of synoviocytes as biological key. Int J Mol Sci 20: 511, 2019.

15. Sharma L: Osteoarthritis year in review 2015: Clinical. Osteoarthritis Cartilage 24: 36-48, 2016.

16. Huynh NPT, Anderson BA, Guilak F and McAlinden A: Emerging roles for long noncoding RNAs in skeletal biology and disease. Connect Tissue Res 58: 116-141, 2017.
17. Tian W, Jiang C, Huang Z, Xu D and Zheng S: Comprehensive analysis of dysregulated lncRNAs, miRNAs and mRNAs with associated ceRNA network in esophageal squamous cell carcinoma. Gene 696: 206-218, 2019.

18. Gaballah HH, Gaber RA, Elrashidy MA, Elshahat DA, Hablus MA and Ebeid AM: Expression of long non-coding RNA CCHE1 in colorectal carcinoma: Correlations with clinicopathological features and ERK/COX-2 pathway. Mol Biol Rep 46: 657-667, 2019

19. Szychlinska MA, Trovato FM, Di Rosa M, Malaguarnera L, Puzzo L, Leonardi R, Castrogiovanni P and Musumeci G: Co-expression and Co-localization of cartilage glycoproteins CHI3L1 and lubricin in osteoarthritic cartilage: Morphological, immunohistochemical and gene expression profiles. Int J Mol Sci 17: 359, 2016.

20. Zhao X, Li J, Lian B, Gu H, Li Y and Qi Y: Global identification of Arabidopsis lncRNAs reveals the regulation of MAF4 by a natural antisense RNA. Nat Commun 9: 5056, 2018.

21. Gardner OF, Musumeci G, Neumann AJ, Eglin D, Archer CW, Alini M and Stoddart MJ: Asymmetrical seeding of MSCs into fibrin-poly(ester-urethane) scaffolds and its effect on mechanically induced chondrogenesis. J Tissue Eng Regen Med 11: 2912-2921, 2017.

22. Wang Q, Wang W, Zhang F, Deng Y and Long Z: NEAT1/miR-181c regulates osteopontin (OPN)-mediated synoviocyte proliferation in osteoarthritis. J Cell Biochem 118: 3775-3784, 2017.

23. Cen X, Huang XQ, Sun WT, Liu Q and Liu J: Long noncoding RNAs: A new regulatory code in osteoarthritis. Am J Transl Res 9: 4747-4755, 2017.

24. Huang H, Zheng J, Shen N, Wang G, Zhou G, Fang Y, Lin J and Zhao J: Identification of pathways and genes associated with synovitis in osteoarthritis using bioinformatics analyses. Sci Rep 8: 10050, 2018.

25. Hayes B, Kittelson A, Loyd B, Wellsandt E, Flug J and Stevens-Lapsley J: Assessing radiographic knee osteoarthritis: An online training tutorial for the kellgren-lawrence grading scale. MedEdPORTAL 12: 10503, 2016.

26. Livak KJ and Schmittgen TD: Analysis of relative gene expression data using real-time quantitative PCR and the 2(-Delta Delta C(T)) method. Methods 25: 402-408, 2001.

27. Yuan J, Yue H, Zhang M, Luo J, Liu L, Wu W, Xiao T, Chen X, Chen $\mathrm{X}$, Zhang $\mathrm{D}$, et al: Transcriptional profiling analysis and functional prediction of long noncoding RNAs in cancer. Oncotarget 7: 8131-8142, 2016.

28. Sun X, Jia B, Qiu XL, Chu HX, Zhang ZQ, Wang ZP and Zhao JJ: Potential functions of long non-coding RNAs in the osteogenic differentiation of human bone marrow mesenchymal stem cells. Mol Med Rep 19: 113-114, 2019.

29. Bian EB, Chen EF, Xu YD, Yang ZH, Tang F, Ma CC, Wang HL and Zhao B: Exosomal lncRNA-ATB activates astrocytes that promote glioma cell invasion. Int J Oncol 54: 713-721, 2019.

30. Wang $\mathrm{X}, \mathrm{Bao} \mathrm{K}$, Wu P, Yu X, Wang C, Ji L and Hong M: Integrative analysis of lncRNAs, miRNAs, and mRNA-Associated ceRNA network in an atopic dermatitis recurrence model. Int J Mol Sci 19: 3263, 2018.

31. Zhang Y, Xu YZ, Sun N, Liu JH, Chen FF, Guan XL, Li A, Wang F, Zhao QF, Wang HY, et al: Long noncoding RNA expression profile in fibroblast-like synoviocytes from patients with rheumatoid arthritis. Arthritis Res Ther 18: 227, 2016.

32. Chen L, Zhang Y, Rao Z, Zhang J and Sun Y: Integrated analysis of key mRNAs and lncRNAs in osteoarthritis. Exp Ther Med 16: 1841-1849, 2018

33. Boehme KA and Rolauffs B: Onset and progression of human osteoarthritis-can growth factors, inflammatory cytokines, or differential miRNA expression concomitantly induce proliferation, ECM degradation, and inflammation in articular cartilage? Int J Mol Sci 19: 2282, 2018.

34. Dolcino M, Pelosi A, Fiore PF, Patuzzo G, Tinazzi E, Lunardi C and Puccetti A: Long non-coding RNAs play a role in the pathogenesis of psoriatic arthritis by regulating MicroRNAs and genes involved in inflammation and metabolic syndrome. Front Immunol 9: 1533, 2018.

35. Yan ZF, Zhao XY, Liu W and Liu XP: UCA1 impacts progress of rheumatoid arthritis by inducing the apoptosis of fibroblast-like synoviocyte. Eur Rev Med Pharmacol Sci 22: 914-920, 2018.

36. Pearson MJ, Philp AM, Heward JA, Roux BT, Walsh DA, Davis ET, Lindsay MA and Jones SW: Long intergenic noncoding RNAs mediate the human chondrocyte inflammatory response and are differentially expressed in osteoarthritis cartilage. Arthritis Rheumatol 68: 845-856, 2016. 
37. Liu Y, Yau MS, Yerges-Armstrong LM, Duggan DJ, Renner JB Hochberg MC, Mitchell BD, Jackson RD and Jordan JM: Genetic determinants of radiographic knee osteoarthritis in African Americans. J Rheumatol 44: 1652-1658, 2017.

38. Xu D, Jiang Y, Yang L, Hou X, Wang J, Gu W, Wang X, Liu L, Zhang $\mathrm{J}$ and $\mathrm{Lu} \mathrm{H}$ : Long noncoding RNAs expression profile and functional networks in rheumatoid arthritis. Oncotarget 8: 95280-95292, 2017.

39. Tanaka N, Ikeda Y, Yamaguchi T, Furukawa H, Mitomi H, Nakagawa T, Tohma S and Fukui N: $\alpha 5 \beta 1$ integrin induces the expression of noncartilaginous procollagen gene expression in articular chondrocytes cultured in monolayers. Arthritis Res Ther 15: R127, 2013.

40. Zhang Y, Ding J, Xu C, Yang H, Xia P, Ma S and Chen H: rBMSCs/ITGA5B1 promotes human vascular smooth muscle cell differentiation via enhancing nitric oxide production. Int J Stem Cells 11: 168-176, 2018.

41. Choi HJ and Zhu BT: Upregulated cyclin B1/CDK1 mediates apoptosis following 2-methoxyestradiol-induced mitotic catastrophe: Role of Bcl-XL phosphorylation. Steroids 150: 108381, 2019.

42. Collins JA, Arbeeva L, Chubinskaya S and Loeser RF: Articular chondrocytes isolated from the knee and ankle joints of human tissue donors demonstrate similar redox-regulated MAP kinase and akt signaling. Osteoarthritis Cartilage 27: 703-711, 2019.

43. Zhou B, Li Q, Wang J, Chen P and Jiang S: Ellagic acid attenuates streptozocin induced diabetic nephropathy via the regulation of oxidative stress and inflammatory signaling. Food Chem Toxicol 123: 16-27, 2019.

44. Monte E, Rosa-Garrido M, Karbassi E, Chen H, Lopez R, Rau CD, Wang J, Nelson SF, Wu Y, Stefani E, et al: Reciprocal regulation of the cardiac epigenome by chromatin structural proteins $\mathrm{hmgb}$ and Ctcf: Implications for transcriptional regulation. J Biol Chem 291: 15428-15446, 2016.
45. Yang H, Antoine DJ, Andersson U and Tracey KJ: The many faces of HMGB1: Molecular structure-functional activity in inflammation, apoptosis, and chemotaxis. J Leukoc Biol 93: 865-873, 2013.

46. Min J, Feng Q, Liao W, Liang Y, Gong C, Li E, He W, Yuan R and Wu L: IFITM3 promotes hepatocellular carcinoma invasion and metastasis by regulating MMP9 through p38/MAPK signaling. FEBS Open Bio 8: 1299-1311, 2018.

47. Luo X, Huang S, Luo S, Liao H, Wang Y, Deng X, Ma F, Ma CW and Zhou L: Identification of genes underlying the enhancement of immunity by a formula of lentinan, pachymaran and tremelia polysaccharides in immunosuppressive mice. Sci Rep 8: 10082, 2018.

48. Lee HM, Sugino H, Aoki C, Shimaoka Y, Suzuki R, Ochi K, Ochi T and Nishimoto N: Abnormal networks of immune response-related molecules in bone marrow cells from patients with rheumatoid arthritis as revealed by DNA microarray analysis. Arthritis Res Ther 13: R89, 2011.

49. Sucur A, Jajic Z, Artukovic M, Matijasevic MI, Anic B, Flegar D, Markotic A, Kelava T, Ivcevic S, Kovacic N, et al: Chemokine signals are crucial for enhanced homing and differentiation of circulating osteoclast progenitor cells. Arthritis Res Ther 19: 142,2017 